Pacific

Journal of

Mathematics

NONCOMMUTATIVE SUMS OF SQUARES

Scott McCullough ANd Mihai PutinaR 


\title{
NONCOMMUTATIVE SUMS OF SQUARES
}

\author{
Scott McCullough And Mihai Putinar \\ We present a proof of Helton's sum-of-squares theorem based upon a theo- \\ rem of Carathéodory and a Hahn-Banach separation argument.
}

\section{Introduction}

Fix a positive integer $g$, let $\mathscr{F}$ denote the free semigroup on the $2 g$ letters of the alphabet $A=\left\{x_{1}, x_{2}, \ldots, x_{g}, y_{1}, y_{2}, \ldots, y_{g}\right\}$, and let $\mathscr{A}$ denote the free semigroup $\mathbb{C}$-algebra on $A$. An element $p$ of $\mathscr{A}$ is a linear combination of elements from $\mathscr{F}$,

$$
p=\sum p_{w} w
$$

where the sum is finite and $p_{w} \in \mathbb{C}$, and is referred to as a polynomial in $A$. The empty word $\varnothing$ is the multiplicative identity, and 0 , the empty sum, is the additive identity.

Equip $\mathscr{A}$ with the involution ${ }^{*}$ as follows. On letters, $x_{j}^{*}=y_{j}, y_{j}^{*}=x_{j}$; on a word $w=w_{1} \cdots w_{n} \in \mathscr{F}$,

$$
w^{*}=w_{n}^{*} \cdots w_{2}^{*} w_{1}^{*}
$$

and finally, on a polynomial $p$ in $A$ as in (1),

$$
p^{*}=\sum p_{w}^{*} w^{*}
$$

where $p_{w}^{*}$ is the complex conjugate of the complex number $p_{w}$.

Let $\mathscr{B}(\mathscr{H})$ be the space of bounded operators on the complex Hilbert space $\mathscr{H}$. Evaluation at a tuple $X=\left(X_{1}, \ldots, X_{g}\right)$ from $\mathscr{B}(\mathscr{H})$ determines a representation $\pi_{X}: \mathscr{A} \rightarrow \mathscr{B}(\mathscr{H})$ that respects the involution. Explicitly, define $\pi_{X}\left(x_{j}\right)=X_{j}$, $\pi_{X}\left(y_{j}\right)=X_{j}^{*}$, and extend $\pi_{X}$ to an algebra homomorphism. It is evident that $\pi_{X}\left(p^{*}\right)=\pi_{X}(p)^{*}$. We will write $p(X)$ instead of $\pi_{X}(p)$.

The purpose of this note is to give a proof of Helton's sum-of-squares (SoS) theorem [Helton 2002] based upon a theorem of Carathéodory and a Hahn-Banach separation argument. If $r_{j} \in \mathscr{A}$ for $j=1, \ldots, m$, we can consider $p=\sum r_{j}^{*} r_{j}$; for $X$ is a tuple of operators, we have $p(X)=\sum r_{j}(X)^{*} r_{j}(X) \geq 0$, where the notation $T \geq 0$ is used to indicate the Hilbert space operator $T$ is positive semidefinite. Given a nonnegative integer $d$, let $\mathscr{A}_{d}$ denote the set of elements of $\mathscr{A}$ of degree at most $d$, namely those $p$ of the form (1) where the sum is over words of length at most $d$. Let $N(d)$ denote the dimension of $\mathscr{A}_{d}$ (as a $\mathbb{C}$ vector space).

Research supported by NSF grant DMS-0140112 (McCullough) and DMS-0100367 (Putinar). 
Theorem 1.1 (Helton). Let $\mathscr{H}$ be a Hilbert space of dimension $N(d)$ and assume $p \in \mathscr{A}_{d}$ satisfies $p(X) \geq 0$ for all tuples $X=\left(X_{1}, \ldots, X_{g}\right)$ from $\mathscr{B}(\mathscr{H})$.

Then there exist elements $r_{j} \in \mathscr{A}$, for $j=1,2, \ldots, N(d)$, such that $p=\sum r_{j}^{*} r_{j}$.

Remark 1.2. Helton states and proves his theorem over $\mathbb{R}$, rather than $\mathbb{C}$. The interested reader should have no difficulty making the necessary modifications to both the statement and proof of Theorem 1.1 to accommodate real scalars. The approach here can also be adapted to deal with the case of self-adjoint variables where $x_{j}^{*}=x_{j}$ [McCullough 2001].

\section{Carathéodory's Theorem}

Let $\mathscr{C}_{d}$ be the convex hull of $\left\{r^{*} r: r \in \mathscr{A}_{d}\right\}$; it is a subset of $\mathscr{A}_{2 d}$. Carathéodory's theorem asserts that a vector belonging to the convex hull of a system of points in an $n$-dimensional real vector space can be written as a convex combination of at most $n+1$ of these points. The proof is elementary and can be found for instance in [Reznick 1992]. In the same spirit we prove the following more precise decomposition result for the cone $\mathscr{C}_{d}$.

Theorem 2.1. If $p \in \mathscr{C}_{d}$, then there exist $m \leq N(d)$ and $r_{j} \in \mathcal{A}_{d}$, for $j=1,2, \ldots, m$, such that $p=\sum r_{j}^{*} r_{j}$.

Sketch of proof. Let $N=N(d)$. Index $\mathbb{C}^{N}$ and $\mathscr{A}_{d}^{N}$ (the algebraic direct sum of $\mathscr{A}_{d}$ with itself $N$ times) by $\mathscr{F}_{d}$, the elements of $\mathscr{F}$ of length at most $d$. Let $V \in \mathscr{A}_{d}^{N}$ denote the border vector [Helton 2002]; that is, the vector whose $w \in \mathscr{F}_{d}$ entry is $w$ (thought of as a column). Given $r=\sum r_{w} w \in \mathscr{A}_{d}$, let $R$ denote the (column) vector with $w$ entry $r_{w}^{*}$. Then

$$
r=R^{*} V
$$

Since $p \in \mathscr{C}_{d}$, there exist $M$ and $r_{1}, \ldots, r_{M} \in \mathscr{A}_{d}$ such that $p=\sum r_{j}^{*} r_{j}$. Let $R_{j}$ denote the corresponding vectors from $\mathbb{C}^{N}$ and let $Q=\sum R_{j} R_{j}^{*}$. Then $Q \geq 0$ and

$$
p=V^{*} Q V \text {. }
$$

Since $Q$ is positive semidefinite and $N \times N$, there exists vectors $Q_{j} \in \mathbb{C}^{N}$ such that $Q=\sum_{j=1}^{N} Q_{j} Q_{j}^{*}$. Let $q_{j}=Q_{j}^{*} V$ and verify that

$$
p=V^{*} Q V=\sum V^{*} Q_{j} Q_{j}^{*} V=\sum q_{j}^{*} q_{j}
$$

\section{Positive Linear Functionals}

In this section we consider positive linear functionals on $\mathscr{A}_{2 d}$; i.e., functionals $\lambda: \mathscr{A}_{2 d} \rightarrow \mathbb{C}$ such that $\lambda\left(p^{*} p\right)>0$ for all nonzero $p \in \mathscr{A}_{d}$.

Lemma 3.1. Suppose $\lambda: \mathscr{A}_{2 d+2} \rightarrow \mathbb{C}$ is a linear functional. If $\lambda\left(p^{*} p\right)>0$ whenever $p \in \mathscr{A}_{d+1}$ is nonzero, then there exists a Hilbert space $\mathscr{H}$ of dimension $N(d), a$ 
vector $\gamma \in \mathscr{H}$, and a tuple $X$ from $\mathscr{B}(\mathscr{H})$ such that $\langle p(X) \gamma, q(X) \gamma\rangle=\lambda\left(q^{*} p\right)$ for all $p, q \in \mathscr{A}_{d}$.

Proof. Let $\mathscr{K}$ denote the Hilbert space obtained by defining the inner product $\langle p, q\rangle=\lambda\left(q^{*} p\right)$ on $\mathscr{A}_{d+1}$ as in the GNS construction. The hypothesis on $\lambda$ guarantees that there are no null vectors. In particular there is no difficulty in defining the following operators. Let $\mathscr{H}$ denote the span of $\mathscr{A}_{d}$ in $\mathscr{K}$ and let $\mathcal{N}$ denote the orthogonal complement of $\mathscr{H}$. Define $S_{j}$ and $T_{j}$ by $S_{j} p=x_{j} p$ and $T_{j} p=y_{j} p$ if $p \in \mathscr{H}$ and $S_{j} p=0=T_{j} p$ if $p \in \mathcal{N}$. Let $P$ denote the orthogonal projection of $\mathscr{K}$ onto $\mathscr{H}$ and let $X_{j}=P S_{j} P$ and $Y_{j}=P T_{j} P$.

For $p, q \in \mathscr{H}$,

$$
\left\langle X_{j} p, q\right\rangle=\left\langle S_{j} p, q\right\rangle=\left\langle x_{j} p, q\right\rangle=\left\langle p, y_{j} q\right\rangle=\left\langle p, Y_{j} q\right\rangle,
$$

where the third equality results from

$$
\left\langle x_{j} p, q\right\rangle=\lambda\left(q^{*} x_{j} p\right)=\lambda\left(\left(y_{j} q\right)^{*} p\right)=\left\langle p, y_{j} q\right\rangle .
$$

Thus $Y_{j}=X_{j}^{*}$ and therefore $p(X) \varnothing=p$ if $p \in \mathscr{A}_{d}$. Further, if $q$ is also in $\mathscr{A}_{d}$, then $\langle p(X) \varnothing, q(X) \varnothing\rangle=\langle p, q\rangle$.

Finally, since $\mathscr{H}=\mathscr{A}_{d}$ as sets, the dimension of $\mathscr{H}$ is $N(d)$.

Lemma 3.2. There exists a linear functional $\mu: \mathscr{A}_{2 d} \rightarrow \mathbb{C}$ such that $\mu\left(p^{*} p\right)>0$ for all nonzero $p \in \mathscr{A}_{d}$.

Proof. Our construction proceeds by induction. Suppose $\mu_{d}: \mathscr{A}_{2 d} \rightarrow \mathbb{C}$ is a linear functional satisfying $\mu_{d}\left(p^{*} p\right)>0$ for all nonzero $p \in \mathscr{A}_{d}$. Define an extension $\mu_{d+1}: \mathscr{A}_{2 d+2} \rightarrow \mathbb{C}$ of $\mu_{d}$ depending on a choice of $C>0$ as follows. A word $v$ is a square if there is a word $w$ such that $v=w^{*} w$. Let $\mu_{d+1}(v)=\mu_{d}(v)$ if $v$ is a word of length at most $2 d$; let $\mu_{d+1}(v)=0$ if $v$ is a word of length $2 d+1$ or $v$ is a word of length $2 d+2$ but is not a square; and let $\mu_{d+1}(v)=C$ if $v$ is a word of length $2 d+2$ that is a square. Since the form $\langle p, q\rangle_{d}=\mu_{d}\left(q^{*} p\right)$ is (strictly) positive definite on $A_{d}$, there is a choice of $C>0$ such that $\langle p, q\rangle_{d+1}=\mu_{d+1}\left(q^{*} p\right)$ is positive definite on $\mathscr{A}_{d+1}$.

To complete the induction argument, simply observe that $\mu_{0}: \mathscr{A}_{0} \rightarrow \mathbb{C}$ given by $\mu_{0}(c \varnothing)=c$ gets the induction started.

Lemma 3.3. There exists a tuple $X=\left(X_{1}, \ldots, X_{g}\right)$ from $\mathscr{B}\left(\mathbb{C}^{N(d)}\right)$ such that if $p \in \mathcal{A}_{d}$ and $p(X)=0$, then $p=0$.

Proof. Combine Lemma 3.1 and Lemma 3.2.

Key to the proof given here of Theorem 1.1 is the fact that $\mathscr{C}_{d}$ is closed in $\mathscr{A}_{2 d}$. Here we mean closed in some, and hence any, norm of $\mathscr{A}_{2 d}$.

Proposition 3.4. The cone $\mathscr{C}_{d}$ is closed in $\mathscr{A}_{2 d}$. 
Proof. Let $X$ denote the tuple from Lemma 3.3 corresponding to $2 d$. Then $\|p\|_{X}=$ $\|p(X)\|$ defines a norm on $\mathscr{A}_{2 d}$. For $p \in \mathscr{A}_{2 d}$ expressed as in (1), the formula

$$
\|p\|_{2}^{2}=\sum\left|p_{w}\right|^{2}
$$

also defines a norm on $\mathscr{A}_{2 d}$. Since $\mathscr{A}_{2 d}$ is finite dimensional, these norms are equivalent.

Suppose $p_{n} \in \mathscr{C}_{d}$ converges to $p \in \mathscr{A}_{2 d}$. Then $p_{n}(X)$ converges to $p(X)$, so that the sequence $\left\{p_{n}(X)\right\}$ is bounded. In view of Theorem 2.1, for each $n$, there exist $r_{j, n} \in \mathscr{A}_{d}$, with $1 \leq j \leq N(d)$, such that

$$
p_{n}=\sum_{j=1}^{N(d)} r_{j, n}^{*} r_{j, n} .
$$

Evaluating this at $X$, we conclude that each sequence $\left\{r_{j, n}(X)\right\}_{n}$ is bounded, and thus, by passing to a subsequence, there exists $r_{j} \in \mathscr{A}_{d}$ such that $r_{n, j}(X)$ converges to $r_{j}(X)$, for $j=1,2, \ldots, N(d)$. Therefore the sequence $\left\{p_{n}(X)\right\}$ converges to

$$
\sum_{j} r_{j}(X)^{*} r_{j}(X)
$$

\section{Helton's Theorem}

It is now possible to prove Theorem 1.1 by arguing the contrapositive. Accordingly, let $\mathscr{H}$ be a Hilbert space of dimension $N(d)$ and suppose $q \in \mathscr{A}_{d}$ satisfies $q(X) \geq 0$ for all tuples $X$ of matrices acting on $\mathscr{H}$, but $q \notin \mathscr{C}_{d}$.

Note that $\mathscr{C}_{m+k} \cap \mathscr{A}_{m}=\mathscr{C}_{m} \cap \mathscr{A}_{m}$ for all positive integers $k, m$, since terms of top degree cannot cancel. Thus we can regard $q \in \mathscr{A}_{2 d}$ and assume that $q \notin \mathscr{C}_{d+1}$.

Let $\mathscr{A}_{k}^{\text {sa }}$ denote the self-adjoint elements of $\mathscr{A}_{k}$, where $p \in \mathscr{A}_{k}$ is self-adjoint provided $p^{*}=p$. Observe that $\mathscr{C}_{k} \subset \mathscr{A}_{2 k}^{\mathrm{sa}}$ and that $p \in \mathscr{A}_{k}$ implies that $p=$ $\operatorname{Re} p+i \operatorname{Im} p$, where both $\operatorname{Re} p=\frac{1}{2}\left(p+p^{*}\right)$ and $\operatorname{Im} p=\frac{1}{2 i}\left(p-p^{*}\right)$ are in $\mathscr{A}_{k}^{\mathrm{sa}}$. If $q^{*} \neq q$, then, by Lemma 3.3, there is a tuple $X$ of operators on the Hilbert space $\mathscr{H}$ so that $q(X)^{*} \neq q(X)$. Thus it may be assumed that $q$ is self-adjoint.

By Proposition 3.4 and Minkowski's separation theorem (see for instance [Reed and Simon 1980]) there exists a real linear functional $v: \mathscr{A}_{2 d+2}^{\mathrm{sa}} \rightarrow \mathbb{R}$ and a real number $c$ such that $v(q)<c \leq v(p)$ for all $p \in \mathscr{C}_{d+1}$. Since $\mathscr{C}_{d+1}$ is a cone, $c=0$. Define $\Lambda: \mathscr{A}_{2 d+2} \rightarrow \mathbb{C}$ by $\Lambda(p)=v(\operatorname{Re} p)+i v(\operatorname{Im} p)$ and verify that $\Lambda$ is indeed a (complex) linear functional and $\Lambda\left(p^{*} p\right)=v\left(p^{*} p\right) \geq 0$ for $p \in \mathscr{A}_{d+1}$.

Let $\mu$ denote the linear functional from Lemma 3.2. There exists $k>0$ such that $(\Lambda+k \mu)(q)<0$. Let $\lambda=\Lambda+k \mu$. Then $\lambda\left(p^{*} p\right)>0$ for all $p \in \mathscr{A}_{d+1}$. Hence, by Lemma 3.1, there exists a Hilbert space $\mathscr{H}$ of dimension $N(d)$, a vector $\gamma \in \mathscr{H}$, and a tuple $X$ from $\mathscr{B}(\mathscr{H})$ such that $\langle q(X) \gamma, \gamma\rangle=\lambda(q)<0$. A contradiction. 


\section{Acknowledgments}

We thank Bill Helton for his generous help and encouragement in the work detailed in this paper and acknowledge borrowing freely from the ideas in [Helton 2002].

\section{References}

[Helton 2002] J. W. Helton, “'Positive' noncommutative polynomials are sums of squares”, Ann. of Math. (2) 156:2 (2002), 675-694. MR 2003k:12002

[McCullough 2001] S. McCullough, "Factorization of operator-valued polynomials in several noncommuting variables", Linear Algebra Appl. 326:1-3 (2001), 193-203. MR 2002f:47035

[Reed and Simon 1980] M. Reed and B. Simon, Methods of modern mathematical physics, I, 2nd ed., Academic Press, New York, 1980. Functional analysis. MR 85e:46002

[Reznick 1992] B. Reznick, Sums of even powers of real linear forms, Mem. Amer. Math. Soc. 463, Amer. Math. Soc., Providence, RI, 1992. MR 93h:11043

Received November 15, 2002.

SCOTt McCullough

DEPARTMENT OF MATHEMATICS

UNIVERSITY OF FLORIDA

GAINESVILLE, FL 32611-8105

sam@math.ufl.edu

Mihai PUTINAR

DEPARTMENT OF MATHEMATICS

UNIVERSITY OF CALIFORNIA

SANTA BARBARA, CA 93106

mputinar@mail.math.ucsb.edu 